Research Article

\title{
Fault Diagnosis of Rolling Bearing Based on Improved VMD and KNN
}

\author{
Quanbo Lu $\mathbb{D}^{1},{ }^{1}$ Xinqi Shen, ${ }^{2}$ Xiujun Wang, ${ }^{3}$ Mei Li $\mathbb{D}^{1},{ }^{1} \mathrm{Jia} \mathrm{Li}^{1}{ }^{1}$ and Mengzhou Zhang \\ ${ }^{1}$ College of Information Engineering, China University of Geosciences, Beijing 100083, China \\ ${ }^{2}$ China Academy of Information and Communications Technology, Beijing 100191, China \\ ${ }^{3}$ Smart Chip Testing Department, Beijing Software Testing \& QA Center, Beijing 100193, China \\ Correspondence should be addressed to Mei Li; maggieli@cugb.edu.cn
}

Received 7 July 2021; Revised 24 August 2021; Accepted 16 September 2021; Published 4 October 2021

Academic Editor: Jie Chen

Copyright ( 92021 Quanbo Lu et al. This is an open access article distributed under the Creative Commons Attribution License, which permits unrestricted use, distribution, and reproduction in any medium, provided the original work is properly cited.

Variational modal decomposition (VMD) has the end effect, which makes it difficult to efficiently obtain fault eigenvalues from rolling bearing fault signals. Inspired by the mirror extension, an improved VMD is proposed. This method combines VMD and mirror extension. The mirror extension is a basic algorithm to inhibit the end effect. A comparison is made with empirical mode decomposition (EMD) for fault diagnosis. Experiments show that the improved VMD outperforms EMD in extracting the fault eigenvalues. The performance of the new algorithm is proven to be effective in real-life mechanical fault diagnosis. Furthermore, in this article, combining with singular value decomposition (SVD), fault eigenvalues are extracted. In this way, fault classification is realized by K-nearest neighbor $(\mathrm{KNN})$. Compared with EMD, the proposed approach has advantages in the recognition rate, which can accurately identify fault types.

\section{Introduction}

VMD is a novel method to deal with the signals. At present, VMD has shown great achievements in the processing of vibration signal, biological signal, and electrical signal. It has been used for detection, diagnosis, and prediction. In the heart sound signal, Babu et al. [1] adopted VMD to extract the Shannon entropy envelope of the heart beat pulse. The approach can accurately identify the signal features reflecting cardiac abnormalities in ECG. Li et al. [2] proposed an adaptive denoising method based on VMD, which is applied to water supply pipeline leakage location. Compared with EMD, VMD can effectively eliminate modal aliasing and is robust to noise and sampling $[3,4]$.

At present, many scholars use different methods to study the vibration signal of fault bearing [5-11]. In the field of fault diagnosis, some scholars study and apply VMD. Zhang et al. [12] constructed a new technique, which applies VMD to mechanical fault diagnosis. Through failure mechanism analysis, they established the fault model in fast Fourier transform (FFT) and envelope analysis. This approach can successfully diagnose the fault of rolling bearing. Jiang et al. [13] presented an adaptive detection method, which uses VMD to detect early defects in bearings. Yi et al. [14] employed VMD to extract bearing fault characteristics. LV et al. [15] used VMD and multicore vector to realize mechanical fault diagnosis. Compared with the traditional fault diagnosis model, this approach had better performance. Yan et al. [16] adopted VMD to extract hybrid-domain features, identifying fault type with high accuracy. VMD has an important application value for mechanical fault diagnosis [17]. However, the drawback of the abovementioned approaches is that they have the modal aliasing in VMD, which affects the diagnostic performance.

In this paper, a new method is proposed for rolling bearing fault diagnosis. First, a mirror extension to suppress its modal aliasing is used and the improved VMD into bearing fault diagnosis is introduced [18]. Then, combine the improved VMD with SVD to achieve the effective extraction of fault eigenvalues, finally adopt KNN to complete fault classification. The contributions of this paper are presented as follows: 
(1) An improved VMD approach is proposed based on VMD and mirror extension, which inhibits the end effect. Improved VMD makes it effective for fault diagnosis.

(2) Compared with EMD, the proposed approach has an advantage in the recognition rate, which can accurately identify the rolling bearing fault type.

(3) The proposed diagnosis framework is effectively verified by detection of the rolling bearing fault. Results demonstrate that our framework has advantages in extracting the characteristic frequency, which is suitable for the detection of manufacturing systems.

The article is organized as follows. The principles of $\mathrm{VMD}$, mirror extension, and KNN are introduced in Section 2. Section 3 provides a new method and the detailed diagnosis scheme of the proposed approach. Section 4 presents the experimental verification. Section 5 concludes this paper.

\section{Theories}

2.1. VMD. VMD is a new approach of signal processing, which is adaptive and quasi-orthogonal. The original signal is decomposed into several modal components using VMD. Each modal component has limited bandwidth, and it is near the central frequency $w_{k}$ [19]. As presented in equation (1), the constrained variational models are calculated:

$$
\begin{aligned}
& \min _{\left\{u_{k}\right\},\left\{w_{k}\right\}}\left\{\sum_{k}\left\|\partial_{t}\left[\left(\delta(t)+\frac{j}{\pi t}\right) u_{k}(t)\right] e^{-j w_{k} t}\right\|_{2}^{2}\right\} \\
& \text { s.t. } \quad \sum_{k} u_{k}=f,
\end{aligned}
$$

where $\left\{u_{k}\right\}=\left\{u_{1}, \ldots, u_{k}\right\}$ are the narrow-band components and $\left\{w_{k}\right\}=\left\{w_{1}, \ldots, w_{k}\right\}$ represent the central frequencies. $\sum_{k}=\sum_{k=1}^{k}$, where $k$ is the number of decompositions.

In order to find the optimal solution of the constrained variational model, the VMD method uses the quadratic penalty function with good convergence and the Lagrange multiplier $\lambda$ operator with strong constraint ability. Therefore, a Lagrangian function $L$ can be introduced to optimize the constrained variational problem so as to minimize the narrow-band component $u_{k}$ and the central frequency $w_{k}$ obtained in equations (3) and (4). The expression of $L$ is introduced:

$$
L\left(\left\{u_{k}\right\},\left\{w_{k}\right\}, \lambda\right)=\alpha \sum_{k}\left\|\partial_{t}\left[\left(\delta(t)+\frac{j}{\pi t}\right) u_{k}(t)\right] e^{-j w_{k} t}\right\|_{2}^{2}+\left\|f(t)-\sum_{k} u_{k}(t)\right\|_{2}^{2}+\left\langle\lambda(t), f(t)-\sum_{k} u_{k}(t)\right\rangle .
$$

The formula for minimizing the narrow-band component $\widehat{u}^{k}$ is expressed as follows:

$$
\widehat{u}_{k}^{n+1}(w)=\frac{\widehat{f}(w)-\sum_{i \neq k} \widehat{u}_{i}(w)+\widehat{\lambda}(w) / 2}{1+2 \alpha\left(w-w_{k}\right)^{2}} .
$$

The formula for minimizing the central frequency narrow-band component $u_{k}$ is obtained as follows:

$$
w_{k}^{n+1}=\frac{\int_{0}^{\infty} w|\widehat{u}(w)|^{2} \mathrm{~d} w}{\int_{0}^{\infty}|\widehat{u}(w)|^{2} \mathrm{~d} w} .
$$

2.2. KNN. KNN is a nonparametric prediction algorithm. It searches for $k$ most similar eigenvectors in the historical database to predict the future value [20]. The model has simple structure and high computational efficiency.

The KNN classification algorithm is described as follows. When the testing sample (unknown sample) is given, first search the pattern space to find the $K$ training sample closest to the testing sample, that is, K-nearest neighbor, and then calculate the selected K-nearest neighbor. If a class has the maximum number of the nearest neighbors, the testing sample can be determined to the class. Euclidean distance is used to calculate the distance between the testing sample and all training samples. The formula is defined as follows:

$$
d(X, Y)=\sqrt{\sum_{i=1}^{N}\left(x_{i}-y_{i}\right)^{2}}
$$

where $X$ is the testing sample and $Y$ denotes the training sample. Figure 1 is the detailed steps of KNN.

\section{The Proposed Diagnosis Method}

3.1. Improved VMD. The mirror extension assumes that a mirror is placed at both ends of the data. The original data sequence image in the mirror is symmetrical with respect to the mirror and the original data sequence [21]. The original data sequence image and the original data sequence in the two mirrors form a continuous and closed ring without endpoints. The internal data completely determines the upper and lower envelopes of the data, so the end effect is fundamentally avoided [22]. Above the mirror surface is the original data. Below the mirror surface is the extension data. After the operation, only the data processing results above the mirror surface are output.

The purpose of boundary extension is not to provide accurate data beyond the end, but to provide a condition that the data within the end completely determine the envelope. The mirror extension satisfies this condition, so it is an ideal extension algorithm.

When VMD analyzes and processes the fault signal, the error is caused by the influence of external factors on both ends [23-27]. Taking into account the end effect in VMD, a mirror extension is adopted to suppress it. The newly proposed method has an advantage in extracting the characteristic frequency. Figure 2 is the detailed steps of the 


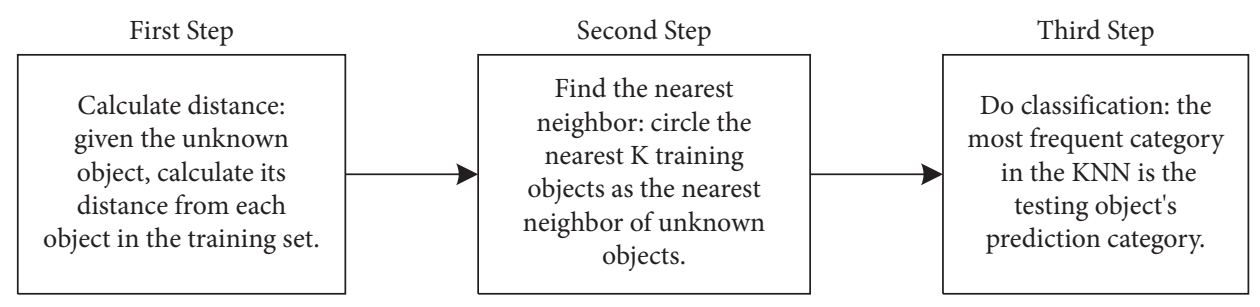

Figure 1: Steps of KNN.

improved VMD. The main process of improved VMD can be summarized as follows:

Step 1 : initialize $\left\{\widehat{u}_{k}^{1}\right\},\left\{w_{k}^{1}\right\}, \hat{\lambda}^{1}, n \longleftarrow 0$, where $\left\{u_{k}\right\}=$ $\left\{u_{1}, \ldots, u_{k}\right\}$ are the narrow-band components, $\left\{w_{k}\right\}=\left\{w_{1}, \ldots, w_{k}\right\}$ represent the central frequencies, $k$ is the number of decompositions, and $\lambda$ represents the Lagrange multiplier.
Step 2 : set $n \longleftarrow n+1$ and $k \longleftarrow k+1$ and execute the whole cycle. Update $\widehat{u}_{k}$ and $w_{k}$ for all $w \geq 0$ to reach the preset decomposition number. When $k=K$, the cycle ends. The updated formula of the narrow-band component and the corresponding central frequency are as follows:

$$
\begin{aligned}
\widehat{u}_{k}^{n+1}(w) & =\frac{\hat{f}(w)-\sum_{i<k} \widehat{u}_{i}^{n+1}(w)-\sum_{i>k} \widehat{u}_{i}^{n}(w)+\hat{\lambda}^{n}(w) / 2}{1+2 a\left(w-w_{k}^{n}\right)^{2}}, \\
\widehat{w}_{k}^{n+1} & =\frac{\int_{0}^{\infty} w\left|\widehat{u}_{k}^{n+1}(w)\right|^{2} \mathrm{~d} w}{\int_{0}^{\infty}\left|\widehat{u}_{k}^{n+1}(w)\right|^{2} \mathrm{~d} w} .
\end{aligned}
$$

Step 3 : update $\lambda$ according to the formula:

$$
\hat{\lambda}^{n+1}(w) \longleftarrow \widehat{\lambda}^{n}(w)+\tau\left(\widehat{f}(w)-\sum_{k} \widehat{u}_{k}^{n+1}(w)\right) .
$$

Step 4 : return to Step 2 and repeat the above process until the whole iterative process meets the constraints, and a series of narrow-band eigenmode component signals are obtained. Equation (9) is the constraint condition, where $\varepsilon$ is set to $10^{-6}$ :

$$
\frac{\sum_{k}\left\|\widehat{u}_{k}^{n+1}-\widehat{u}_{k}^{n}\right\|_{2}^{2}}{\left\|\widehat{u}_{k}^{n}\right\|_{2}^{2}<\varepsilon}
$$

The specific extension process is provided as follows:

(1) Find all extreme points of the rolling bearing fault signal $x(t), t=1,2, \ldots, T$. The extreme points include the local maximum points and the local minimum points.

The local maximum point sequence of the signal $x(t)$ is

$$
\left\{\left(f_{\max }(1), x_{\max }(1)\right),\left(f_{\max }(2), x_{\max }(2)\right), \Lambda\left(f_{\max }\left(t_{\max }\right), x_{\max }\left(t_{\max }\right)\right)\right\} .
$$

The local minimum point sequence of the signal $x(t)$

is

$$
\left\{\left(f_{\min }(1), x_{\min }(1)\right),\left(f_{\min }(2), x_{\min }(2)\right) \Lambda\left(f_{\min }\left(t_{\min }\right), x_{\min }\left(t_{\min }\right)\right)\right\}
$$

where $f_{\max }(t), t=1,2, \ldots, T$, are the local maximum points, $f_{\min }(t), t=1,2, \ldots, T$, are the local minimum points, $x_{\max }(t), t=1,2, \ldots, T$, are the maximum points, and $x_{\min }(t), t=1,2, \ldots, T$, are the minimum points.
(2) According to equation (12), the symmetrical extension is employed to the extreme points of the fault signal $x(t)$ to obtain a new extreme point sequence $\left\{p^{\prime}\right\}$ : 


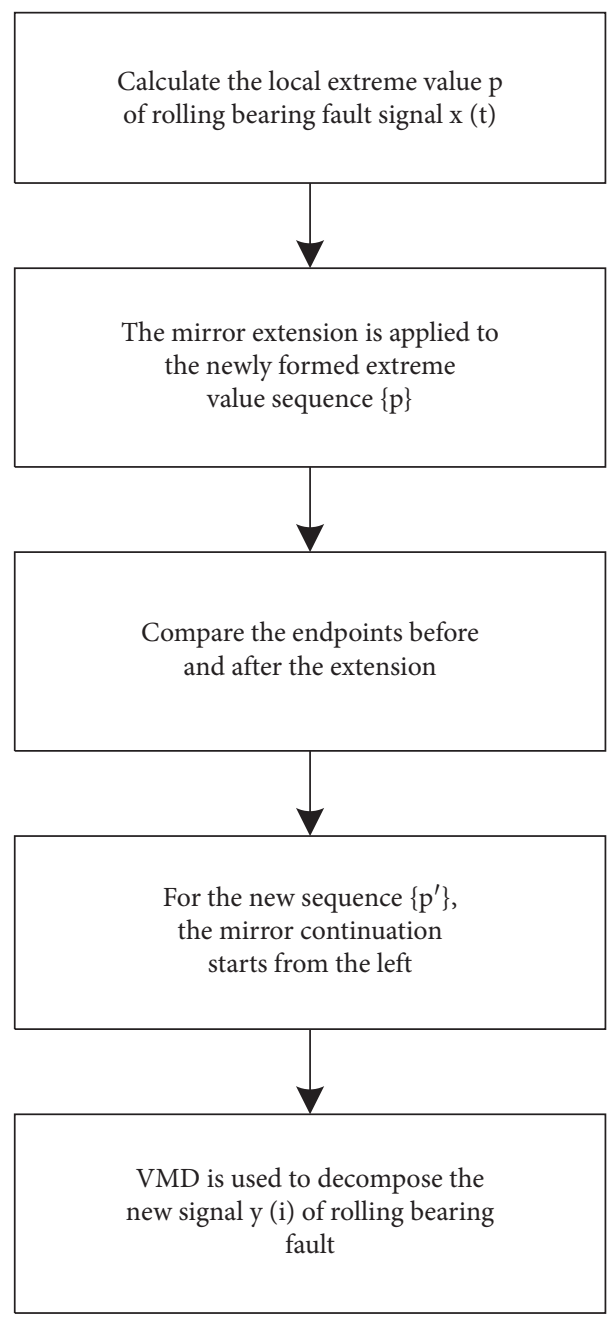

Figure 2: Steps of the improved VMD.

$$
\left\{\begin{array}{l}
\left\{\begin{array}{l}
f_{\max }(0)=f_{\max }(1) \\
x_{\max }(0)=2 * x_{\max }(1)-x_{\max }(2), \text { when } \quad x_{\min }(1)<x_{\max }(1),
\end{array}\right. \\
\left\{\begin{array}{l}
f_{\min }(0)=f_{\min }(1) \\
x_{\min }(0)=2 * x_{\min }(1)-x_{\min }(2), \text { when } \quad x_{\min }(1)>x_{\max }(1),
\end{array}\right. \\
\left\{\begin{array}{l}
f_{\max }\left(t_{\max }+1\right)=f_{\max }\left(t_{\max }\right) \\
x_{\max }\left(t_{\max }+1\right)=2 * x_{\max }\left(t_{\max }\right)-x_{\max }\left(t_{\max }-1\right), \text { when } \quad x_{\min }\left(t_{\min }\right)>x_{\max }\left(t_{\max }\right)
\end{array}\right. \\
\left\{\begin{array}{l}
f_{\min }\left(t_{\min }+1\right)=f_{\min }\left(t_{\min }\right) \\
x_{\min }\left(t_{\min }+1\right)=2 * x_{\min }\left(t_{\min }\right)-x_{\min }\left(t_{\min }-1\right)
\end{array}, \text { when } \quad x_{\min }\left(t_{\min }\right)<x_{\max }\left(t_{\max }\right) .\right.
\end{array}\right.
$$

(3) Correct the new extreme point sequence $\left\{p^{\prime}\right\}$ after extension:

$$
\left\{\begin{array} { l } 
{ f _ { \operatorname { m i n } } ( 0 ) > x ( 1 ) , \text { when } \quad x _ { \operatorname { m a x } } ( 1 ) < x _ { \operatorname { m i n } } ( 1 ) } \\
{ f _ { \operatorname { m a x } } ( 0 ) > x ( 1 ) , \text { when } \quad x _ { \operatorname { m a x } } ( 1 ) > x _ { \operatorname { m i n } } ( 1 ) }
\end{array} \left\{\begin{array}{l}
f_{\min }\left(t_{\min }\right)>x(t) \text {, when } \quad x_{\min }\left(t_{\min }\right)<x_{\max }\left(t_{\max }\right) \\
f_{\max }\left(t_{\max }\right)<x(t) \text {, when } \quad x_{\min }\left(t_{\min }\right)>x_{\max }\left(t_{\max }\right)
\end{array} .\right.\right.
$$

(4) Use the value on the left end of the fault signal $x(t)$ as the symmetry plane, and the mirror extension is realized. Meanwhile, the new fault signal $y(i)$ of rolling bearing is realized. 
3.2. Diagnosis Scheme. Owing to the feature extraction is difficult in the incipient rolling bearing failures, this paper presents a new method combined improved VMD and KNN. The structure of the diagnosis scheme is shown in Figure 3.

The detailed steps of the diagnosis scheme are as follows:

(1) Firstly, improved VMD is applied to handle the fault signal, and several narrow-band eigenmode components $u$ are realized.

(2) Then, the narrow-band components are selected, which have rich fault information. These modal components are selected to reconstruct the fault signal, and the envelope spectrum is conducted for it.

(3) Finally, the feature vector from modal components is extracted by using SVD. After feature extraction, there are 100 data sets. 70 of the 100 data sets are randomly employed to train the KNN classification model, and the rest are employed to test the classification performance.

\section{Experimental Verification}

4.1. Experimental Setting. As shown in Figure 4, the fault diagnosis platform consists of a $0.75 \mathrm{~kW}$ three-phase asynchronous motor, two couplings, a reducer, a magnetic powder brake, a piezoelectric accelerometer, a faulty rolling bearing, and a photoelectric speed sensor. The faulty rolling bearing is installed in the bearing pedestal of the reducer.

The signal acquisition system is used to collect the fault signals, in which the rotation speed $n$ and the sampling frequency $f$ are set at $600 \mathrm{r} / \mathrm{min}$ and $1 \mathrm{kHz}$, respectively. Table 1 is the basic parameters of rolling bearing. According to these parameters, the fault characteristic frequencies are calculated: inner race is $99 \mathrm{~Hz}$ and outer race is $71 \mathrm{~Hz}$. The improved VMD is applied to decompose the fault signal. When the mode number $K$ is different, their central frequency is different. The relationship between them is depicted in Figure 5.

4.2. Experimental Results and Analysis. When the value of $K$ starts from 5, the central frequency is close [26], see Figure 5(a). This is an over decomposition phenomenon. Hence, the $K$ value taken in the test is 5. Based on VMD experience, the balance parameter constrained by data fidelity adopts the default value of 2000, and the time step of the double rise is 0.1 . Figure 6(a) illustrates the time domain diagram of the inner race fault, and its improved VMD result is demonstrated in Figure 7(a).

As illustrated in Figure 5(b), when the value of $K$ starts from 5 , the central frequency is close, which is an over decomposition phenomenon [28]. Thereby, the $K$ value taken in the test is 5. Based on VMD experience, the balance parameter constrained by data fidelity adopts the default value of 2000, and the time step of the double rise is 0.1 . Figure 6(b) shows the time domain diagram of the outer race fault. The improved VMD result is demonstrated in Figure 7(b).
As shown in Figure 6(a), the original signal of the inner race fault contains considerable background noise, which obscures the fault information. As shown in Figure 7(a), compared with the original signal, the modal components after the improved VMD eliminate the noise, which is the function of Wiener filter. There are obvious fault shock components in $U_{3}, U_{4}$, and $U_{5}$. Therefore, these three modal components are selected to reconstruct the signal, and the envelope spectrum is conducted for it. The result of the envelope spectrum is depicted in Figure 8(a).

In contrast to the improved VMD, the first five components of EMD are also taken, and Figure 9(a) is the decomposition results. In Figure 9(a), IMF $1, \mathrm{IMF}_{2}$, and $\mathrm{IMF}_{3}$ contain many fault feature information, and they are reconstructed. Figure 8(b) presents the envelope spectrum of the reconstructed signal.

From Figure 6(b), the original signal time domain of the outer race fault contains large background noise, which obscures the fault information. Figure 7 (b) shows that the modal components eliminate the noise, which is the function of the Wiener filter. There are obvious fault shock components in $U_{2}, U_{4}$, and $U_{5}$. Hence, these three modal components are selected to reconstruct the signal, and the envelope spectrum is conducted for it. The result of the envelope spectrum is provided in Figure 10(a).

As a comparative test analysis, the first five components of EMD are taken, and Figure 9(b) is the decomposition result. As evident in Figure 9(b), $\mathrm{IMF}_{1}, \mathrm{IMF}_{2}$, and $\mathrm{IMF}_{3}$ contain many fault feature information, and they are reconstructed. Figure 10(b) presents the envelope spectrum of the reconstructed signal.

Figure 8(a) displays that the envelope spectrum has obvious fault shock characteristics around $99 \mathrm{~Hz}$, which is basically consistent with the theoretical value. The small gap does not affect the fault identification. $198 \mathrm{~Hz}$ is the second frequency, and $297 \mathrm{~Hz}$ is the third frequency. As shown in Figure 8(b), the envelope spectrum is not ideal. $99 \mathrm{~Hz}$ and its octave are not demodulated. Consequently, the decomposition result of the improved VMD outperforms EMD.

Figure 10(a) shows that the envelope spectrum has obvious fault shock characteristics around $71 \mathrm{~Hz}$. This is basically consistent with the theoretical value. The small gap does not affect the fault identification. $142 \mathrm{~Hz}$ is the second frequency, and $213 \mathrm{~Hz}$ is the third frequency. In Figure 10(b), the envelope spectrum is not ideal. $71 \mathrm{~Hz}$ and its octave are not demodulated. Therefore, the decomposition result of the improved VMD is shown to outperform EMD.

In the fault classification, $K$ in the $\mathrm{KNN}$ algorithm is set to 1 . The first three groups of singular values are selected as the nearest neighbor classification training sets and testing sets. For each fault type, 70 of the 100 datasets are randomly employed to train the KNN classification model, and the rest are applied to test the classification performance. The fault classification results after improved VMD + SVD are shown in Figure 11(a), and the fault classification results after EMD + SVD are provided in Figure 11(b). Figures 11(a) and 11(b) show that the performance of the improved VMD + SVD is better than EMD + SVD. 


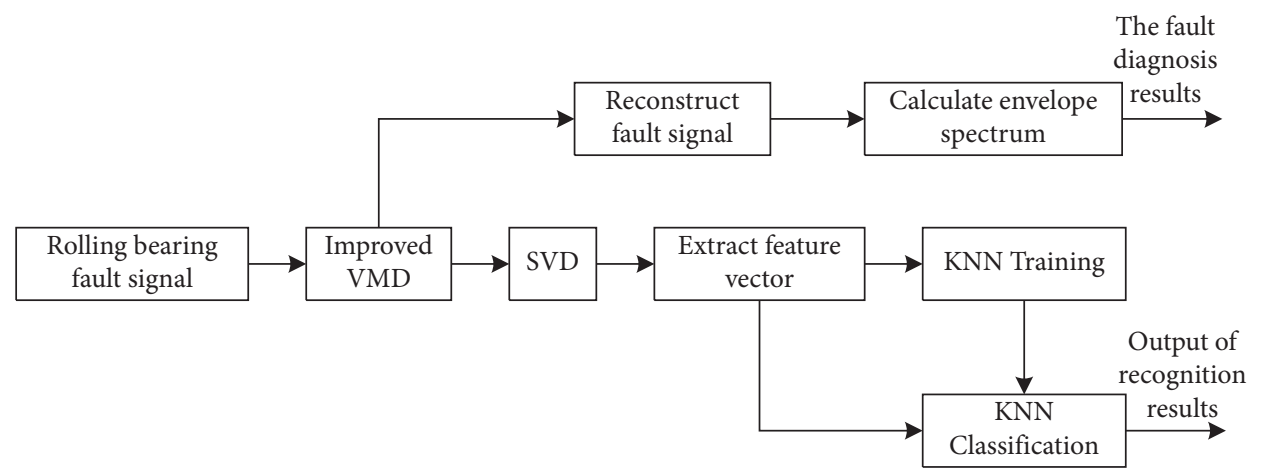

FIgURE 3: The structure of the diagnosis scheme.

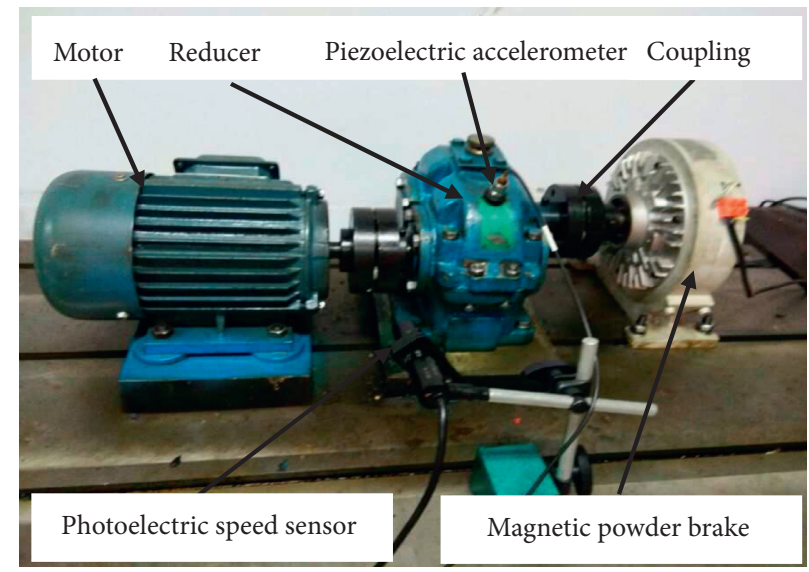

FIgURE 4: The fault diagnosis platform.

TABle 1: The parameters of bearing.

\begin{tabular}{lccc}
\hline The diameter of angle rolling elements & Bearing pitch & The number of rolling elements & Contact angle \\
\hline 8 & 46 & 17 & $14^{\circ}$ \\
\hline
\end{tabular}
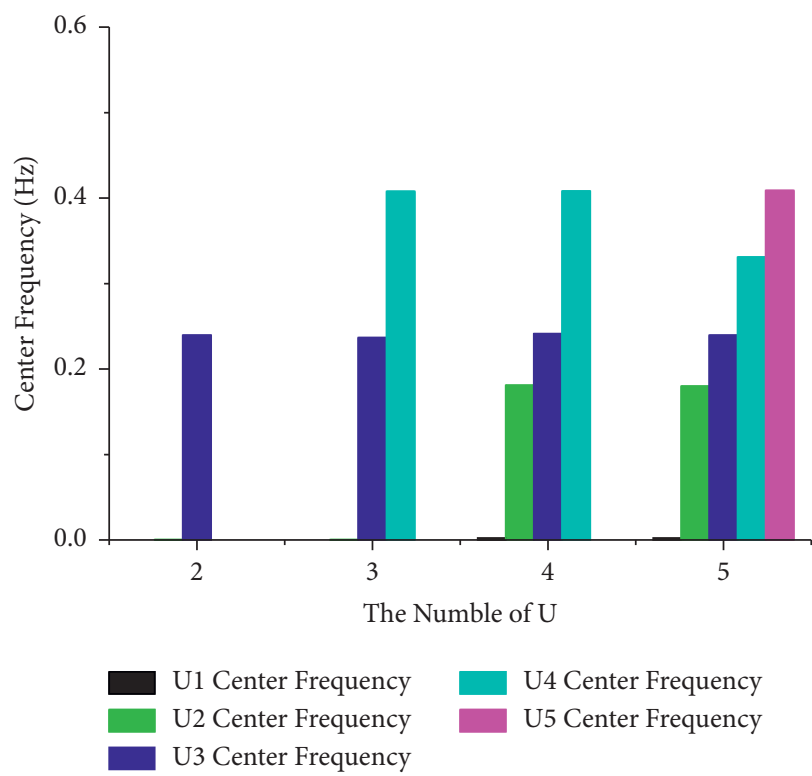

(a)
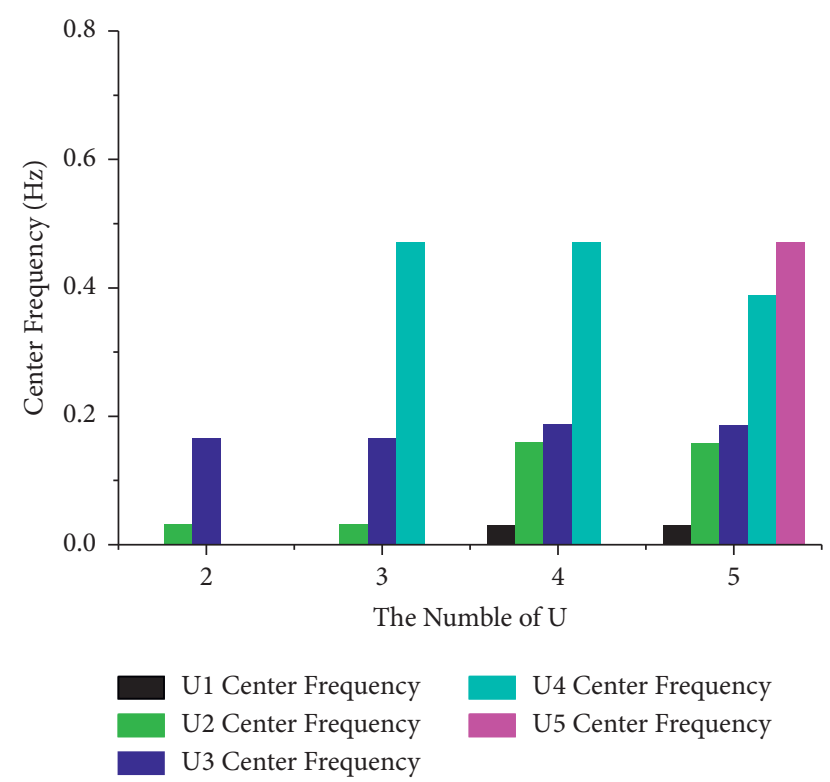

(b)

FIGURE 5: The relationship between mode number and central frequency after improved VMD. (a) Inner race fault. (b) Outer race fault. 


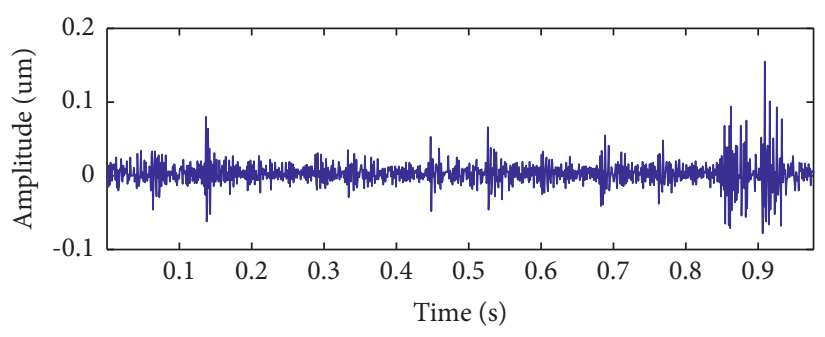

(a)

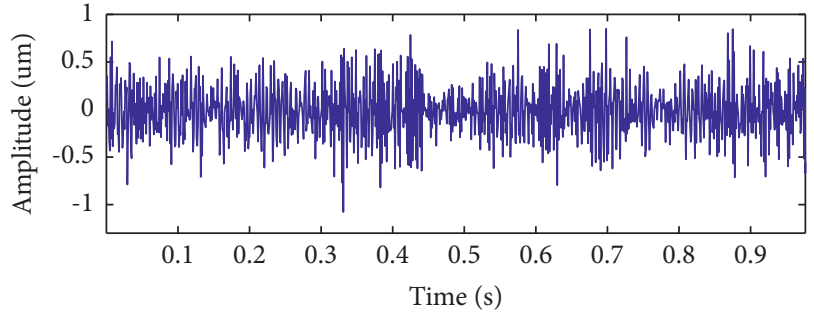

(b)

FIgURE 6: Time domain diagram. (a) Inner race fault. (b) Outer race fault.
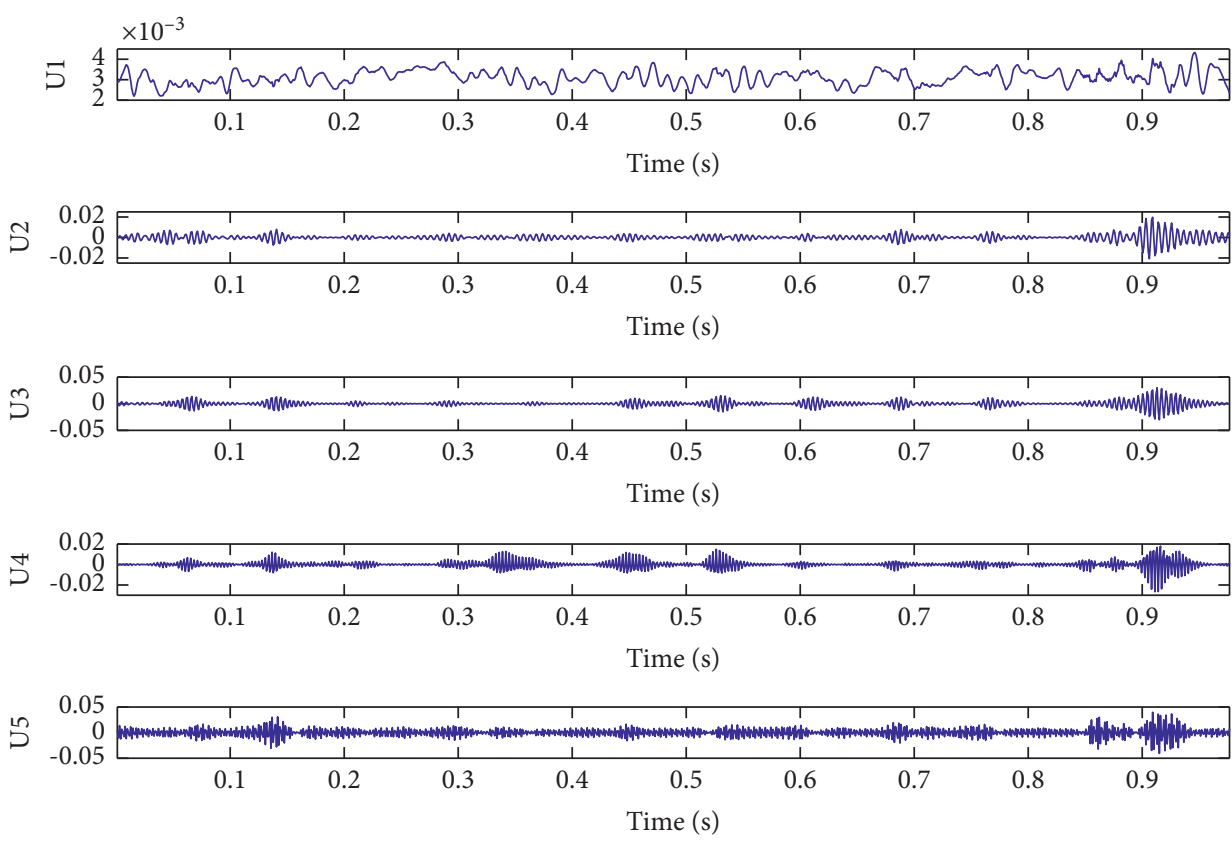

(a)
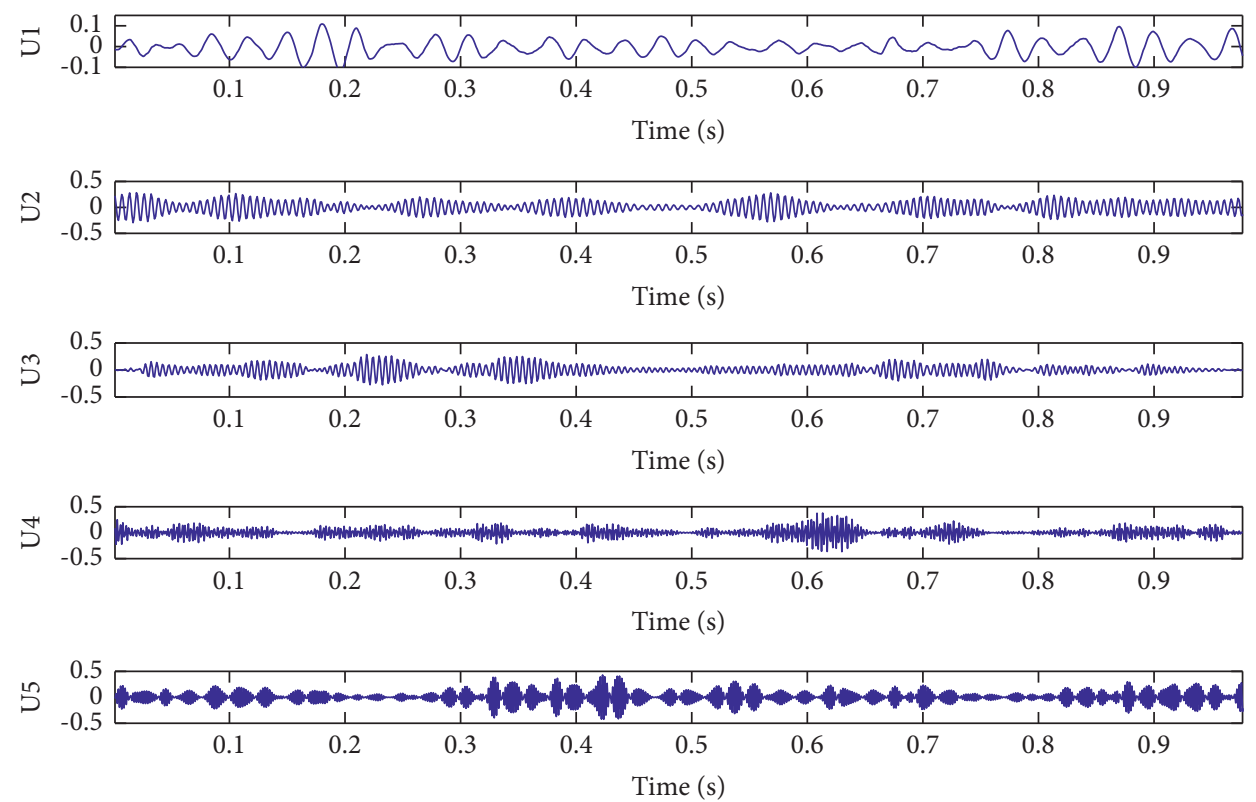

(b)

Figure 7: The decomposition results after improved VMD. (a) Inner race fault. (b) Outer race fault. 


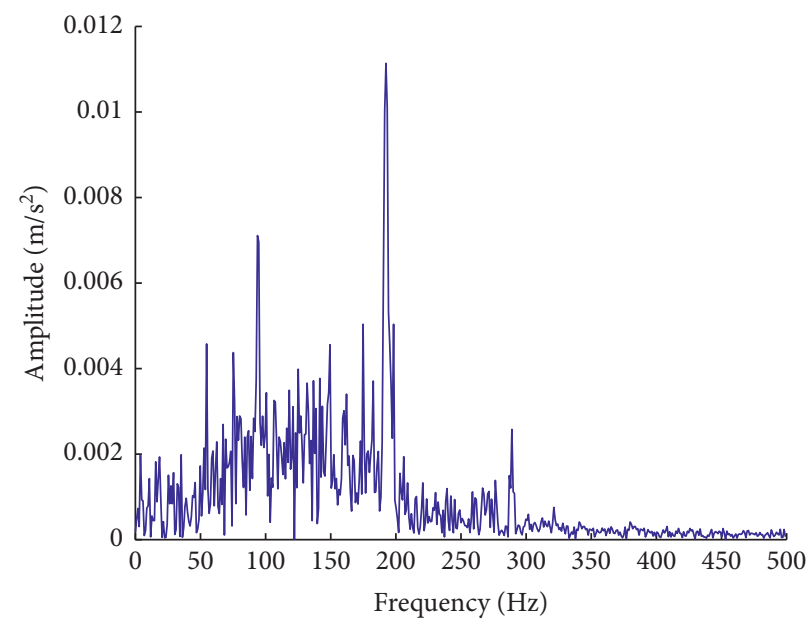

(a)

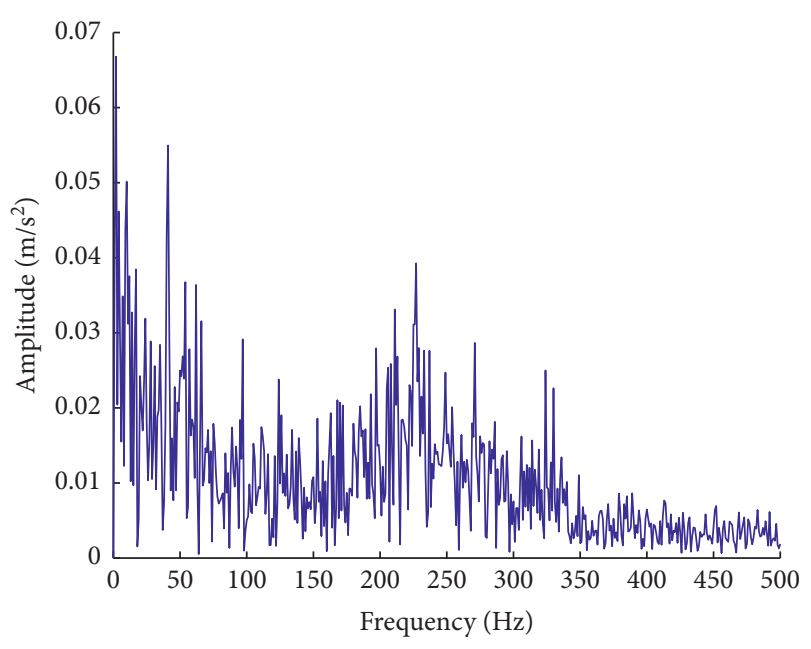

(b)

FIGURE 8: Envelope spectrum of inner race fault. (a) $U_{3}+U_{4}+U_{5}$. (b) $\mathrm{IMF}_{1}+\mathrm{IMF}_{2}+\mathrm{IMF}_{3}$.
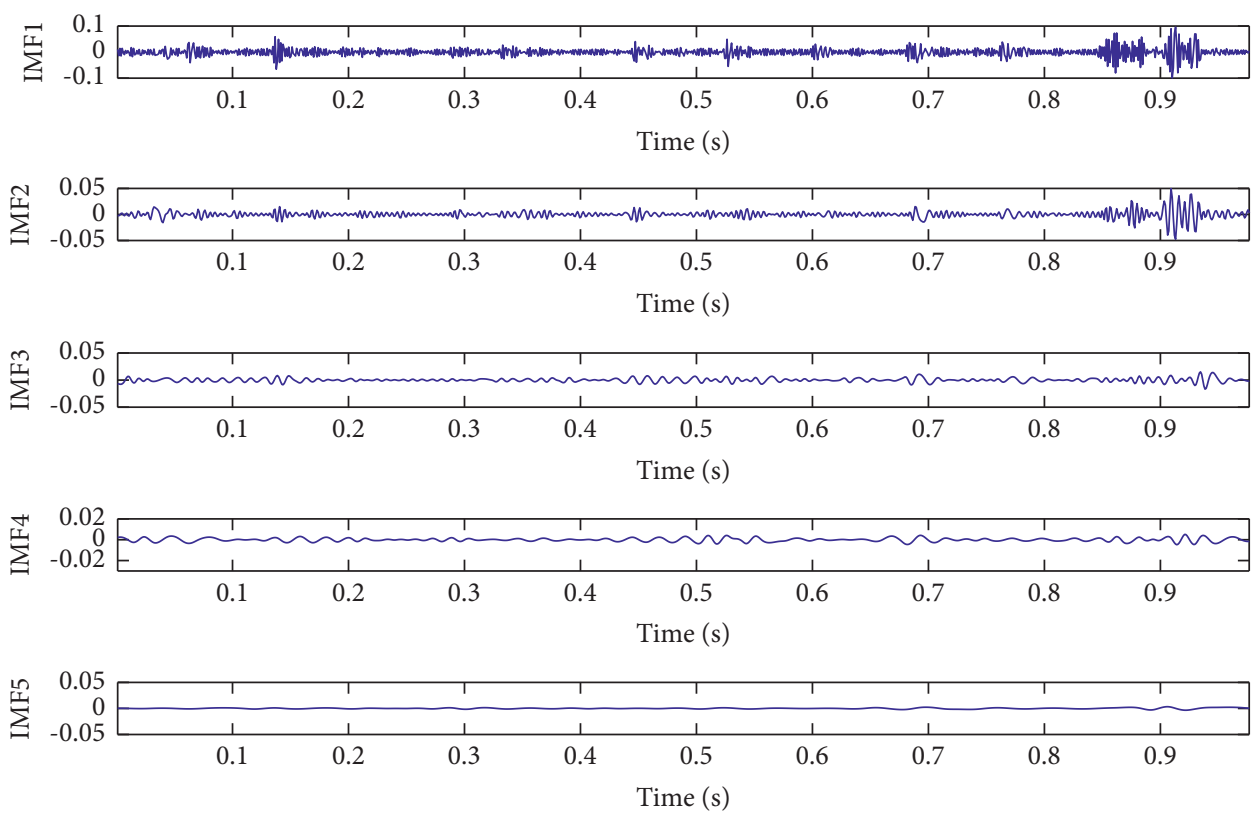

(a)

Figure 9: Continued. 

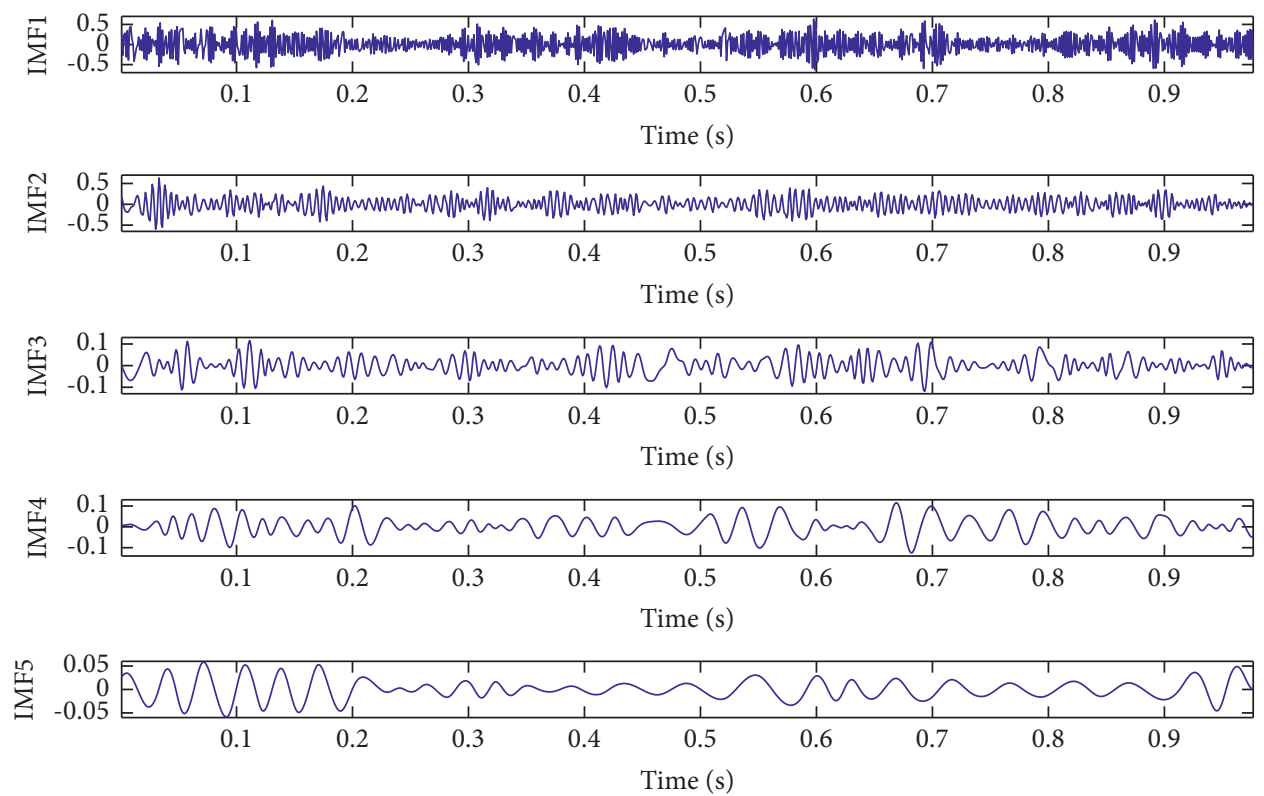

(b)

Figure 9: The decomposition results after EMD. (a) Inner race fault. (b) Outer race fault.

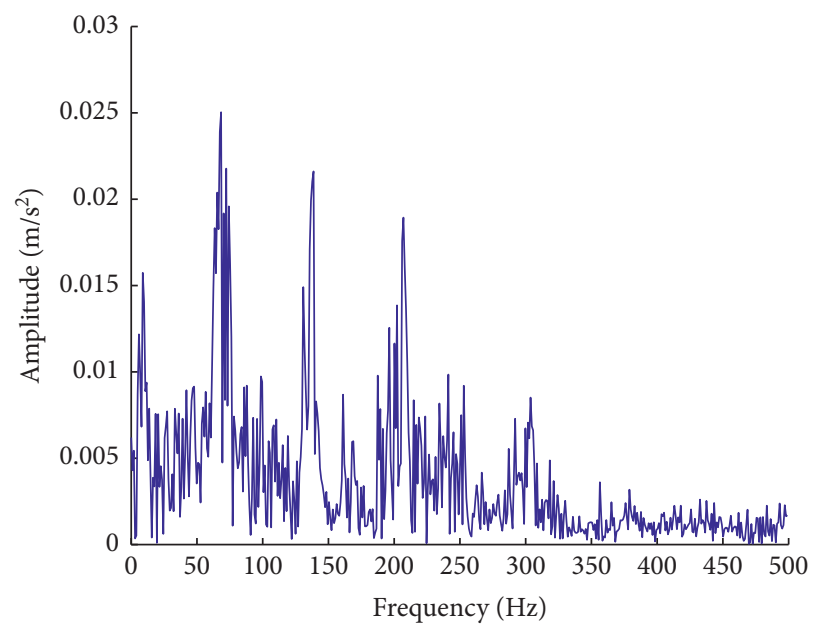

(a)

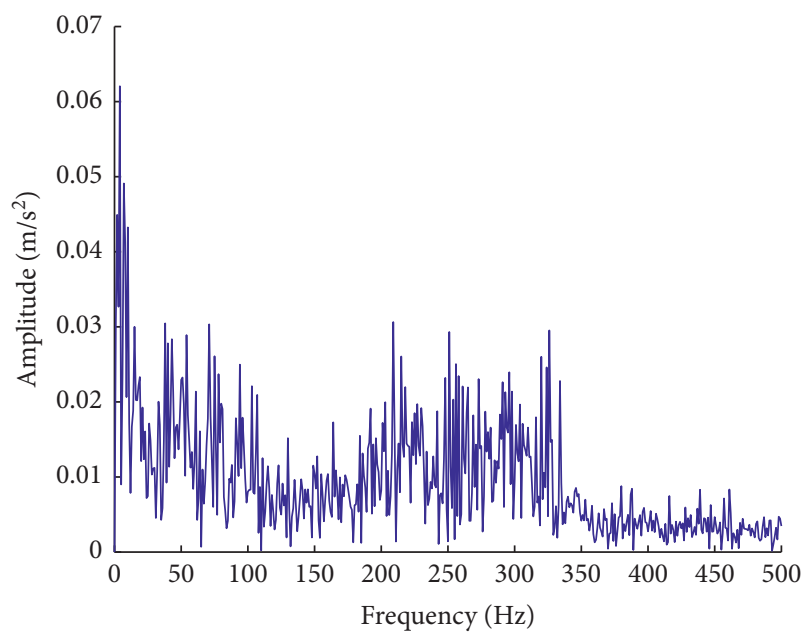

(b)

Figure 10: Envelope spectrum of outer race fault. (a) $U_{2}+U_{4}+U_{5}$. (b) $I M F_{1}+I M F_{2}+I_{M F}$.

The fault recognition rates are reported in Table 2. In the classification results after the improved VMD + SVD, one inner race fault is incorrectly identified as the outer race fault, and one outer race fault is incorrectly identified as the inner race fault. In the classification results after EMD + SVD, three inner race faults are incorrectly identified as the outer race faults, and five outer race faults are incorrectly identified as the inner race faults. 


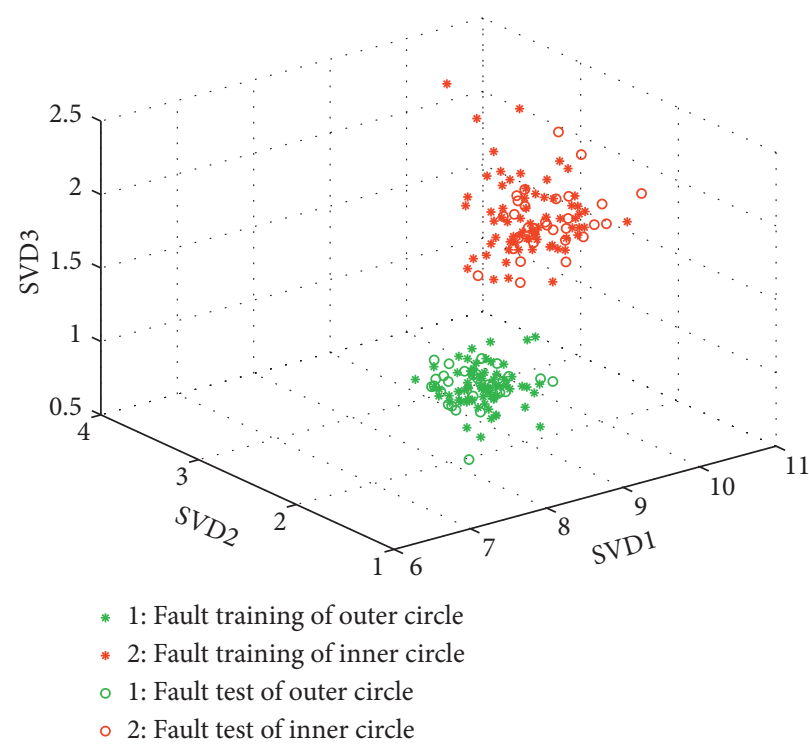

(a)

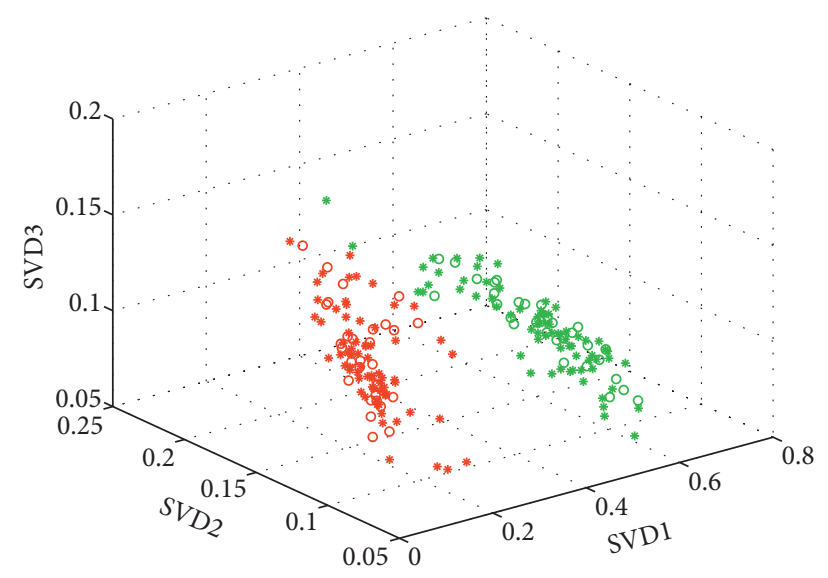

* 1: Fault training of outer circle

* 2: Fault training of inner circle

- 1: Fault test of outer circle

- 2: Fault test of inner circle

(b)

FIgURE 11: Fault classification result. (a) Improved VMD + SVD and (b) EMD + SVD.

TABle 2: The fault recognition rates.

\begin{tabular}{lccc}
\hline Algorithm & Inner race fault & Outer race fault & Recognition rate (\%) \\
\hline Improved VMD + SVD & 29 & 29 & 96.67 \\
EMD + SVD & 27 & 25 & 86.67 \\
\hline
\end{tabular}

\section{Conclusion}

A new method combining the improved VMD and KNN for the rolling bearing fault detection system is presented. First, the improved VMD decomposes the fault signal to obtain the modal component. Then, the modal components with many fault shock components are reconstructed. Finally, the envelope spectrum is applied to the reconstructed signal, and the characteristic frequency is identified successfully. Furthermore, combining with SVD, KNN realizes fault type classification. The experimental results demonstrate that the proposed approach has potential application in rolling bearing fault diagnosis.

\section{Data Availability}

Some or all data, models, or code generated or used during the study are available from the corresponding author upon request.

\section{Conflicts of Interest}

The authors have no conflicts of interest/competing interest to disclose.

\section{Acknowledgments}

This work was supported by the National Natural Science Foundation of China (Grant no. 41572347).

\section{References}

[1] K. A. Babu, B. Ramkumar, and M. S. Manikandan, "Automatic identification of S1 and S2 heart sounds using simultaneous PCG and PPG recordings," IEEE Sensors Journal, vol. 18, no. 22, pp. 9430-9440, 2018.

[2] J. Li, Y. Chen, Z. Qian, and C. Lu, "Research on VMD based adaptive denoising method applied to water supply pipeline leakage location," Measurement, vol. 151, pp. 1-13, 2020.

[3] A. Dibaj, M. Mohammad Ettefagh, R. Hassannejad, and M. Biuok Ehghaghi, "A hybrid fine-tuned VMD and CNN scheme for untrained compound fault diagnosis of rotating machinery with unequal-severity faults," Expert Systems with Applications, vol. 11, pp. 1-16, 2020.

[4] A. Joshuva, R. S. Kumar, S. Sivakumar, G. Deenadayalan, and R. Vishnuvardhan, "An insight on VMD for diagnosing wind turbine blade faults using C4.5 as feature selection and discriminating through multilayer perceptron," Alexandria Engineering Journal, vol. 59, no. 5, pp. 3863-3879, 2020.

[5] W. Deng, H. Liu, J. Xu, H. Zhao, and Y. Song, "An improved quantum-inspired differential evolution algorithm for deep belief network," IEEE Transactions on Instrumentation and Measurement, vol. 69, no. 10, pp. 7319-7327, 2020.

[6] H. M. Zhao, S. Y. Zuo, M. Hou et al., "A novel adaptive signal processing method based on enhanced empirical wavelet transform technology," Sensors, vol. 18, pp. 1-17, 2018.

[7] M. Kuncan, "An intelligent approach for bearing fault diagnosis: combination of 1D-LBP and GRA," IEEE Access, vol. 8, pp. 137517-137529, 2020.

[8] K. Zhang, Y. Xu, Z. Liao, L. Song, and P. Chen, "A novel fast entrogram and its applications in rolling bearing fault 
diagnosis," Mechanical Systems and Signal Processing, vol. 154, Article ID 107582, 2021.

[9] J. Cheng, Y. Yang, X. Li, and J. Cheng, "Adaptive periodic mode decomposition and its application in rolling bearing fault diagnosis," Mechanical Systems and Signal Processing, vol. 161, Article ID 107943, 2021.

[10] X. Wang, D. Mao, and X. Li, "Bearing fault diagnosis based on vibro-acoustic data fusion and $1 \mathrm{D}-\mathrm{CNN}$ network," Measurement, vol. 173, Article ID 108518, 2021.

[11] J. Wei, H. Huang, L. Yao, Y. Hu, Q. Fan, and D. Huang, "New imbalanced bearing fault diagnosis method based on Samplecharacteristic oversampling technique (SCOTE) and multiclass LS-SVM," Applied Soft Computing, vol. 101, Article ID 107043, 2021.

[12] M. Zhang, Z. Jiang, and K. Feng, "Research on variational mode decomposition in rolling bearings fault diagnosis of the multistage centrifugal pump," Mechanical Systems and Signal Processing, vol. 93, pp. 460-493, 2017.

[13] X. Jiang, S. Li, and C. Cheng, "A novel method for adaptive multiresonance bands detection based on VMD and using MTEO to enhance rolling element bearing fault diagnosis," Shock and Vibration, vol. 2016, Article ID 8361289, 20 pages, 2016.

[14] C. Yi, Y. Lv, and Z. Dang, "A fault diagnosis scheme for rolling bearing based on particle swarm optimization in variational mode decomposition," Shock and Vibration, vol. 2016, Article ID 9372691, 10 pages, 2016.

[15] Z. Lv, B. Tang, Y. Zhou, and C. Zhou, "A novel method for mechanical fault diagnosis based on variational mode decomposition and multikernel support vector machine," Shock and Vibration, vol. 201611 pages, 2016.

[16] X. Yan, Y. Liu, M. Jia, and Y. Zhu, “A multi-stage hybrid fault diagnosis approach for rolling element bearing under various working conditions," IEEE Access, vol. 7, pp. 138426-138441, 2019.

[17] X. An and J. Yang, "Denoising of hydropower unit vibration signal based on variational mode decomposition and approximate entropy," Transactions of the Institute of Measurement and Control, vol. 38, no. 3, pp. 282-292, 2016.

[18] F. Jiang, Z. Zhu, and W. Li, "An improved VMD with empirical mode decomposition and its application in incipient fault detection of rolling bearing," IEEE Access, vol. 6, pp. 44483-44493, 2018.

[19] K. Dragomiretskiy and D. Zosso, "Variational mode decomposition," IEEE Transactions on Signal Processing, vol. 62, no. 3, pp. 531-544, 2014.

[20] N. Ling, G. Aneiros, and P. Vieu, "kNN estimation in functional partial linear modeling," Statistical Papers, vol. 61, no. 1, pp. 423-444, 2020.

[21] C. Dong, X. Jiao, and F. Xu, "Mirror extensions of vertex operator algebras," Communications in Mathematical Physics, vol. 329, no. 1, pp. 263-294, 2014

[22] J. Wang, W. Liu, and S. Zhang, "An approach to eliminating end effects of EMD through mirror extension coupled with support vector machine method," Personal and Ubiquitous Computing, vol. 23, no. 3-4, pp. 443-452, 2019.

[23] M. G. A. Nassef, T. M. Hussein, and O. Mokhiamar, "An adaptive variational mode decomposition based on sailfish optimization algorithm and gini index for fault identification in rolling bearings," Measurement, vol. 108514, pp. 1-20, 2020.

[24] S. Braut, R. Žigulić, A. Skoblar, and G. Štimac Rončević, "Partial rub detection based on instantaneous angular speed measurement and variational mode decomposition," Journal of Vibration Engineering \& Technologies, vol. 8, no. 2, pp. 351-364, 2020.

[25] Z. Wang, J. Wang, and W. Du, "Research on fault diagnosis of gearbox with improved variational mode decomposition," Sensors, vol. 18, no. 10, p. 3510, 2018.

[26] Y. Miao, M. Zhao, and J. Lin, "Identification of mechanical compound-fault based on the improved parameter-adaptive variational mode decomposition," ISA Transactions, vol. 84, pp. 82-95, 2019.

[27] Y. X. Wang, L. Yang, and J. W. Xiang, "A hybrid approach to fault diagnosis of roller bearings under variable speed conditions," Measurement Science and Technology, vol. 4, pp. 1587-1601, 2017.

[28] H. Liu, D. Li, Y. Yuan, S. Zhang, H. Zhao, and W. Deng, "Fault diagnosis for a bearing rolling element using improved VMD and HT," Applied Sciences, vol. 9, no. 7, p. 1439, 2019. 